\title{
Associations of night-time road traffic noise with carotid intima-media thickness and blood pressure : The Whitehall II and SABRE study cohorts
}

\author{
Halonen, Jaana I.
}

2017-01

Halonen , J I , Dehbi , H-M , Hansell , A L , Gulliver , J , Fecht , D , Blangiardo , M , Kelly , F J , Chaturvedi , N , Kivimaki , M \& Tonne , C 2017 , ' Associations of night-time road traffic noise with carotid intima-media thickness and blood pressure : The Whitehall II and SABRE study cohorts ' , Environment International , vol. 98 , pp. 54-61 . https://doi.org/10.1016/j.envint.2016.09.023

http://hdl.handle.net/10138/230301

https://doi.org/10.1016/j.envint.2016.09.023

publishedVersion

Downloaded from Helda, University of Helsinki institutional repository.

This is an electronic reprint of the original article.

This reprint may differ from the original in pagination and typographic detail.

Please cite the original version. 


\title{
Associations of night-time road traffic noise with carotid intima-media thickness and blood pressure: The Whitehall II and SABRE study cohorts
}

\author{
Jaana I. Halonen a ${ }^{\mathrm{a}, 1,2}$, Hakim-Moulay Dehbi ${ }^{\mathrm{b}, *, 1}$, Anna L. Hansell ${ }^{\mathrm{a}, \mathrm{c}}$, John Gulliver ${ }^{\mathrm{a}}$, Daniela Fecht ${ }^{\mathrm{a}}$, \\ Marta Blangiardo ${ }^{\mathrm{a}}$, Frank J. Kelly ${ }^{\mathrm{d}}$, Nish Chaturvedi ${ }^{\mathrm{e}}$, Mika Kivimäki ${ }^{\mathrm{f}, \mathrm{g}}$, Cathryn Tonne ${ }^{\mathrm{h}, \mathrm{i}, \mathrm{j}}$
}

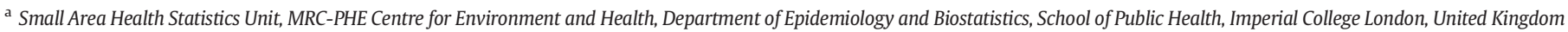

${ }^{b}$ Department of Epidemiology and Biostatistics, Imperial College London, London, United Kingdom

c Public Health and Primary Care, Imperial College Healthcare NHS Trust, London, UK

${ }^{d}$ MRC-PHE Centre for Environment and Health, King's College London, United Kingdom

e Institute of Cardiovascular Science, University College London, London, United Kingdom

${ }^{\mathrm{f}}$ Department of Epidemiology and Public Health, University College London, London, United Kingdom

g Clinicum, Faculty of Medicine, University of Helsinki, Helsinki, Finland

h ISGlobal, Centre for Research in Environmental Epidemiology (CREAL), Barcelona, Spain

${ }^{\mathrm{i}}$ Universitat Pompeu Fabra (UPF), Spain

j CIBER Epidemiología y Salud Pública (CIBERESP), Spain

\section{A R T I C L E I N F O}

\section{Article history:}

Received 1 July 2016

Received in revised form 7 September 2016

Accepted 27 September 2016

Available online 3 October 2016

\section{Keywords:}

Cardiovascular

Cohort study

Epidemiology

Hypertension

Traffic noise

\begin{abstract}
A B S T R A C T
Background: Road traffic noise has been linked to increased risk of stroke, for which hypertension and carotid intima-media thickness (cIMT) are risk factors. A link between traffic noise and hypertension has been established, but there are few studies on blood pressure and no studies on cIMT.

Objectives: To examine cross-sectional associations for long-term exposure to night-time noise with cIMT, systolic blood pressure (SBP), diastolic blood pressure (DBP) and hypertension.

Methods: The study population consisted of 2592 adults from the Whitehall II and SABRE cohort studies living within Greater London who had cIMT, SBP and DBP measured. Exposure to night-time road traffic noise (Aweighted $\mathrm{dB}$, referred to as $\mathrm{dBA}$ ) was estimated at each participant's residential postcode centroid.

Results: Mean night-time road noise levels were $52 \mathrm{dBA}(\mathrm{SD}=4)$. In the pooled analysis adjusted for cohort, sex, age, ethnicity, marital status, smoking, area-level deprivation and NOx there was a $9.1 \mu \mathrm{m}(95 \% \mathrm{CI}:-7.1,25.2)$ increase in cIMT in association with $10 \mathrm{dBA}$ increase in night-time noise. Analyses by noise categories of 55$60 \mathrm{dBA}(16.2 \mu \mathrm{m}, 95 \% \mathrm{Cl}:-8.7,41.2)$, and > $60 \mathrm{dBA}(21.2 \mu \mathrm{m}, 95 \% \mathrm{Cl}:-2.5,44.9)$ vs. $<55 \mathrm{dBA}$ were also positive but non-significant, expect among those not using antihypertensive medication and exposed to $>60 \mathrm{dBA}$ vs. $<55 \mathrm{dBA}(32.6 \mu \mathrm{m}, 95 \% \mathrm{CI}: 6.2,59.0)$. Associations for SBP, DPB and hypertension were close to null.

Conclusions: After adjustments, including for air pollution, the association between night-time road traffic noise and cIMT was only observed among non-medication users but associations with blood pressure and hypertension were largely null.
\end{abstract}

(c) 2016 Elsevier Ltd. All rights reserved.

\section{Introduction}

There is growing interest in the potential link between exposure to traffic noise and various cardiovascular health outcomes (Babisch, 2014; Babisch et al., 2014; Barcelo et al., 2016; Bodin et al., 2016; Munzel et al., 2014, 2016; Stansfeld, 2015). We recently reported an area-level association between road traffic noise and increased risk of

\footnotetext{
* Corresponding author at: 67 Isobel Place, N15 4FP London, United Kingdom. E-mail addresses: jaana.halonen@ttl.fi (J.I. Halonen), hakdehbi@gmail.com (H.-M. Dehbi).

1 Co-first authors.

2 Current address: PO Box 70, 70101 Kuopio, Finland.
}

strokes in London, UK (Halonen et al., 2015b). The observed association is biologically plausible based on an established link between noise exposure and hypertension (van Kempen et al., 2012), a major risk factor for stroke (Elliott et al., 2005). Increasing carotid intima-media thickness (cIMT) reflects changes in the vascular walls due to plaque formation as part of the atherosclerotic process. These changes, when severe, increase the risk of occlusion that may result in acute atherothrombotic events such as myocardial infarction and stroke (Eikendal et al., 2015; O'Leary et al., 1999). Although associations between air pollution and cIMT have been investigated (Perez et al., 2015; Tonne et al., 2012; Wilker et al., 2013), we are not aware of studies examining associations between exposure to road traffic noise and cIMT. This association could be on the stress-related pathway between traffic noise exposure and 
cardiovascular events (Basner et al., 2014; Munzel et al., 2014) as systolic blood pressure stress reactions, for example, have been linked to progression of cIMT (Jennings et al., 2004).

In this cross-sectional study, we examined whether long-term exposure to night-time road traffic noise is associated with higher cIMT and blood pressure or higher odds of hypertension among participants of the Whitehall II (WHII) cohort study of British civil servants and the participants of tri-ethnic population-based Southall and Brent REvisited (SABRE) cohort study who lived within Greater London in 2002-2012. We hypothesized that higher road noise exposure at the participants' residential postcodes is associated with higher cIMT, higher blood pressure and higher odds of hypertension.

\section{Methods}

\subsection{Study population}

The study population consisted of participants of two UK cohorts: participants included in the phase 7 clinic visit (2002-2004) of the WHII study of civil servants in England, (Marmot et al., 2005) and participants of the phase 2 (2008-2012) clinic visit of the SABRE tri-ethnic population-based cohort (Tillin et al., 2012). We included participants from both cohorts who had cIMT and blood pressure measured, lived within Greater London and whose residential postcode was available for noise exposure linkage, a total of 3270 adults. Residential addresses were not available due to confidentiality. After excluding those with missing data on any of the covariates the analytical sample size was 2592. The University College London Medical School Committee on the Ethics of Human Research and NHS Health Research Authority NRES London-Harrow Committee (ref. 85/0938) provided ethical approval for the Whitehall II study, and written informed consent was obtained from all participants. Approval for the SABRE study at baseline was obtained from Ealing, Hounslow and Spelthorne, and University College London research ethics committees, and at follow-up from St Mary's Hospital Research Ethics Committee (ref. 07/H0712/109).

\subsection{Carotid intima-media thickness}

For the participants of the WHII cohort in Phase 7 clinical examination, ultrasound vascular measures were performed at the Vascular Physiology Unit, Institute of Child Health, London UK. Measurements were taken in a temperature controlled (22-26 degrees centigrade), quiet room using a non-invasive, high- resolution ultrasound system, the Aloka 5500 with a $7.5 \mathrm{MHz}$ transducer. Participants were examined in a supine position, with the head turned to a 45 degree angle away from the side to be scanned. Intima-media thickness was measured in the right and left common carotid arteries. Longitudinal images of the common carotid artery, triggered on the R-wave of the ECG, were magnified and recorded in DICOM format as a cine loop, on the hard drive of the ultrasound machine for later analysis. The common cIMT was measured at its thickest part $1 \mathrm{~cm}$ proximal to the bifurcation. A measurement was taken between the leading edge of the intima and the media adventitia on three separate images on each side using electronic calipers and the mean of the six measures was used for analysis.

For all participants of the SABRE cohort who attended for a clinic visit, cIMT was measured by an experienced sonographer at St Mary's hospital in London, UK, using an iE33 ultrasound machine with a 7.5 MHz transducer (Philips UK Ltd., Guildford, UK) and concurrent recording of 3-lead ECG. Participants were in supine position on the scan bed. The distal $1 \mathrm{~cm}$ of the (left and right) common carotid artery was imaged after identifying the plane containing the bifurcation of the carotid bulb into the internal and external carotid arteries. Two additional angles, approximately 45 degrees anteriorly and posteriorly, were also used. Three to five cardiac cycles were stored as cine loops for analysis.

\subsection{Blood pressure}

In the WHII study, blood pressure was measured at the same clinic visit as CIMT. SBP and DBP were measured twice in the sitting position after five minutes of rest with an Omron HEM 907 digital blood pressure monitor (Omron Healthcare, Inc., Bannockburn, Illinois). The average of the two readings was taken to be the measured SBP and DBP (Kivimaki et al., 2009).

Blood pressure of the SABRE cohort participants was measured at St Mary's hospital in London, UK. Resting SBP and DBP were measured three times using an Omron 705IT (Omron Healthcare, Inc., Bannockburn, Illinois). For both SBP and DBP, the average of the second and third measurements was retained as the measured SBP and DBP.

Because $34 \%$ of the study sample had medication for hypertension (Table 1), we adjusted their measurement for SBP with an additional $10 \mathrm{mmHg}$, and DBP with an additional $5 \mathrm{mmHg}$ (Cui et al., 2003).

\subsection{Hypertension}

Use of hypertension medication was requested in the study questionnaires in both cohorts. For the analyses hypertension was defined as taking hypertension medication and/or having SBP $>140$ and $\mathrm{DBP}>90$.

\subsection{Road traffic noise}

Annual, A-weighted road traffic noise levels (all $\mathrm{dB}$ are A-weighted and hereafter referred to as dBA) for the years 2003-2009 were modelled at geometric centroids of the 2592 participants' residential

Table 1

Characteristics of the study participants included in the analysis.

\begin{tabular}{|c|c|c|c|c|}
\hline \multirow[b]{3}{*}{ Variable } & \multirow{3}{*}{$\begin{array}{l}\frac{\text { All participants }}{*} \\
\text { Pooled } \\
n=3270\end{array}$} & \multicolumn{3}{|c|}{ Included participants } \\
\hline & & \multirow{2}{*}{$\frac{\text { Pooled }}{n=2592}$} & \multirow{2}{*}{$\frac{\text { WHII }}{n=1965}$} & \multirow{2}{*}{$\begin{array}{l}\text { SABRE } \\
\mathrm{n}=627\end{array}$} \\
\hline & & & & \\
\hline \multicolumn{5}{|l|}{ Outcomes } \\
\hline $\mathrm{SBP}($ mean $(\mathrm{sd}))$ [mmHg] & $135.3(20.4)$ & $133.1(19.8)$ & $128.4(17.6)$ & $147.5(19.4)$ \\
\hline DBP (mean (sd)) [mmHg] & $76.4(10.9)$ & $75.7(11.0)$ & $74.2(10.8)$ & $80.7(10.0)$ \\
\hline $\operatorname{cIMT}($ mean $(\mathrm{sd}))[\mu \mathrm{m}]$ & 840 (192.9) & $823.2(180.8)$ & $791.6(155.9)$ & $922.3(214.6)$ \\
\hline \multicolumn{5}{|l|}{ Covariates } \\
\hline Age (mean (sd)) [years] & $64.1(7.4)$ & $62.8(7.0)$ & $60.8(5.9)$ & $69.4(6.2)$ \\
\hline Sex $=$ male $(\%)$ & $2235(68.3)$ & $1800(69.4)$ & 1315 (66.9) & $485(77.4)$ \\
\hline Hypertension treatment $=$ yes $(\%)$ & $1311(40.1)$ & $890(34.3)$ & $460(23.4)$ & $430(68.6)$ \\
\hline Marital status = non-married (\%) & $804(25.8)$ & $682(26.3)$ & $471(24.0)$ & $211(33.7)$ \\
\hline Smoking = current smoker (\%) & $251(7.7)$ & $199(7.7)$ & $154(7.8)$ & $45(7.2)$ \\
\hline Ethnicity = non-Caucasian (\%) & $975(29.8)$ & $593(22.9)$ & $269(13.7)$ & $324(51.7)$ \\
\hline
\end{tabular}

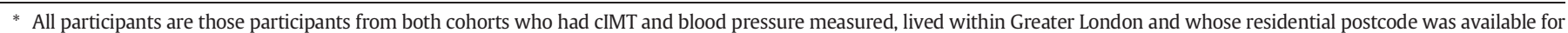
noise exposure linkage. Included participants are those among all participants without missing data on any of the covariates. 
postcode locations using the TRAffic Noise EXposure (TRANEX) model with $0.1 \mathrm{dBA}$ noise level resolution (Gulliver et al., 2015). As the geometric centroid of the postcode falls on a building, we moved all centroids to $1 \mathrm{~m}$ from the facade facing the road. TRANEX uses detailed information on traffic for $\sim 63,000$ road links including varying flows and speeds for each study year, and detailed information on land cover and heights of individual buildings. Validation studies conducted in two UK cities showed Spearman's correlation between modelled and measured noise levels to be high: 0.90 ( $p$-value $<0.0001$ ), suggesting good model performance (Gulliver et al., 2015).

Exposures to day- and night-time noise were estimated. Due to the high correlation of 0.99 between day- and night-time road noise, we chose to focus on night-time noise only, as potential exposure misclassification is reduced in night-time when the majority of people are more likely to be at home. We report health effects of night-time road noise independently from day-time road noise as suggested by WHO (2011). For the TRANEX model, night-time exposure was $\mathrm{L}_{\text {night, }}$ which is an average of hourly measures from 23:00 to 6:59. For the categorical analyses, night-time road noise estimates were categorized by $5 \mathrm{dBA}$ increments: $<55$ (reference), 55-60, and $>60 \mathrm{dBA}$, like in our previous work (Halonen et al., 2015b). We also conducted supplementary analyses using daytime road noise ( $\mathrm{L}_{\text {Aeq16 hour, }}$ from 7:00 to 22:59) and report the effects independently of night-time road noise. For the WHII participants we linked noise level from year 2003 or 2004, whichever was closer to each participant's clinical measurements. For the SABRE participants noise levels from 2009 were used.

\subsection{Covariates}

Data for the individual-level covariates were obtained from the study questionnaires and clinic visits. The following individual-level variables were used in the analyses: sex, age, ethnicity (categorized as white vs. other), marital status (married/cohabiting vs. other) and smoking status (current smoker vs. other). In addition to sex and age, we included smoking and ethnicity a priori given they are strongly associated with cardiovascular health (Martin et al., 2015; Messner et al., 2014), and may plausibly show spatial variability that could be associated with that of noise, given highly diverse composition of the London population, the spatial variability of ethnic groups within London and different smoking prevalences between different ethnic and social groups. Of other individual-level variables available, only marital status was found to be predictive of cIMT and associated with noise exposure and was not a potential intermediate, satisfying the definition of a confounder. In SABRE, ethnicity was described at baseline by the interviewer based on appearance and parental origin. In WHII, self-reported ethnicity at phase 5 was mainly used; missing data were complemented by observer-assigned ethnicity from phase 1 . Marital status and smoking status were based on responses to questionnaires in both cohorts. Other possible individual-level confounders such as body mass index, alcohol drinking and physical activity were not available in both cohorts and thus could not be included in the analyses.

To adjust for traffic-related air pollution like in prior studies (Monrad et al., 2016; Sorensen et al., 2011), annual nitrogen oxide (NOx) concentrations within WHII participants' postcode centroids were estimated for 2003 and 2004 using a dispersion model (Beevers et al., 2013). NOx estimates for the SABRE participants were for year 2009 and modelled for each participant's postcode using a Land Use Regression (LUR) model developed as part of the European Study of Cohorts for Air Pollution Effects (ESCAPE) (Beelen et al., 2013). NOx was weakly associated with exposure, although not predictive of outcomes. To control for possible confounding by area deprivation we used the Index of Multiple Deprivation (IMD). Lower Layer Super Output Area (LSOA) IMD was assigned to postcodes within the LSOA (Office for National Statistics, 2014). Information from 2004 (Noble et al., 2004) was linked to the WHII participants and from 2010 (McLennan et al., 2011) to the SABRE participants. In the analyses we used continuous IMD scores, where higher values indicate greater deprivation.

\subsection{Statistical analyses}

We used linear regression models to examine pooled associations for night-time road traffic noise with cIMT, SBP, and DBP, and logistic regression models for hypertension. Results for these analyses are presented in two complementary ways: 1) using noise as a continuous variable, as $\mu \mathrm{m}$ change in cIMT and as $\mathrm{mmHg}$ change in SBP and DBP per $10 \mathrm{dBA}$ increase in noise level, 2) using noise as categorical variable ( $<55 \mathrm{dBA}$ (reference), 55-60 dBA, and $>60 \mathrm{dBA}$ ), as mean differences in cIMT (in $\mu \mathrm{m}$ ), and SBP and DBP (in $\mathrm{mmHg}$ ) between the reference category and the other two categories. For hypertension, we present odds ratios (OR) and 95\% confidence intervals (CI). We repeated these analyses using daytime road traffic noise.

To examine the effects of adjustments for individual- and area-level covariates on the associations under study we used three different models: Model 1 was adjusted for cohort, age and sex; Model 2 in addition to Model 1 for individual-level ethnicity, marital status and smoking status; and Model 3 in addition to Model 2 for area-level deprivation and NOx. Adjustment for cohort was needed to account for differences in how the outcomes and NOx were measured. To explore potential differences in effect we ran sensitivity analysis including only those not taking anti-hypertensive medications. We also stratified

Table 2

Descriptive statistics of the environmental variables used in the pooled and cohort-specific analyses.

\begin{tabular}{|c|c|c|c|c|c|c|c|}
\hline Environmental variables & Minimum & 10th percentile & Median & Mean & 90th percentile & Maximum & SD \\
\hline \multicolumn{8}{|l|}{ Night-time noise (dBA) } \\
\hline Pooled & 49.2 & 49.3 & 49.8 & 51.7 & 59.2 & 75.4 & 4.3 \\
\hline WH II & 49.2 & 49.3 & 49.7 & 51.4 & 58.4 & 75.4 & 4.0 \\
\hline SABRE & 49.2 & 49.3 & 50.3 & 52.7 & 61.4 & 74.0 & 4.9 \\
\hline \multicolumn{8}{|l|}{ Daytime noise (dBA) } \\
\hline Pooled & 54.8 & 54.9 & 55.3 & 57.2 & 64.4 & 78.1 & 4.1 \\
\hline WH II & 54.8 & 54.9 & 55.2 & 56.9 & 63.5 & 78.1 & 3.8 \\
\hline SABRE & 54.8 & 54.9 & 55.7 & 58.1 & 66.3 & 77.5 & 4.7 \\
\hline \multicolumn{8}{|l|}{$\operatorname{NOx}\left(\mu \mathrm{g} / \mathrm{m}^{3}\right)$} \\
\hline Pooled & 25.0 & 44.4 & 57.1 & 60.0 & 78.2 & 227.5 & 15.7 \\
\hline WH II & 36.1 & 47.2 & 60.7 & 62.5 & 79.8 & 143.4 & 13.2 \\
\hline SABRE & 25.0 & 39.9 & 48.3 & 52.1 & 63.7 & 227.5 & 19.7 \\
\hline \multicolumn{8}{|l|}{$\mathrm{IMD}^{\mathrm{a}}$} \\
\hline Pooled & 1.0 & 5.1 & 15.5 & 18.2 & 35.2 & 70.4 & 12.3 \\
\hline WH II & 1.0 & 4.4 & 12.1 & 15.5 & 31.8 & 70.4 & 11.0 \\
\hline SABRE & 2.97 & 12.3 & 25.0 & 26.7 & 44.3 & 62.3 & 12.2 \\
\hline
\end{tabular}

a $\mathrm{IMD}=$ Index of Multiple Deprivation standardised using all Lower Layer Super Output Areas in England. Higher value indicates higher area-level deprivation. 
Table 3

Pooled and cohort-specific associations for $10 \mathrm{dBA}$ increase in night-time noise with cIMT, SBP and DBP.

\begin{tabular}{|c|c|c|c|c|c|c|c|c|c|}
\hline \multirow[b]{2}{*}{ Outcome } & \multicolumn{3}{|l|}{ Model $1^{\mathrm{a}}$} & \multicolumn{3}{|l|}{ Model $2^{\text {b }}$} & \multicolumn{3}{|l|}{ Model 3c } \\
\hline & Estimate & $95 \% \mathrm{CI}$ & & Estimate & $95 \% \mathrm{CI}$ & & Estimate & $95 \% \mathrm{CI}$ & \\
\hline \multicolumn{10}{|l|}{$\operatorname{cIMT}(\mu \mathrm{m})$} \\
\hline Pooled & 9.8 & -5.0 & 24.7 & 8.0 & -6.9 & 22.8 & 9.1 & -7.1 & 25.2 \\
\hline SABRE & 6.6 & -26.0 & 39.1 & 4.9 & -27.8 & 37.5 & 14.3 & -22.6 & 51.1 \\
\hline WHII & 12.4 & -4.1 & 28.8 & 11.5 & -4.9 & 27.9 & 8.8 & -8.7 & 26.4 \\
\hline \multicolumn{10}{|c|}{$\mathrm{SBP}(\mathrm{mmHg})$} \\
\hline Pooled & 0.1 & -1.5 & 1.7 & 0.0 & -1.6 & 1.6 & 0.3 & -1.4 & 2.1 \\
\hline SABRE & -0.5 & -3.6 & 2.5 & -1.3 & -4.3 & 1.7 & -1.1 & -4.5 & 2.3 \\
\hline WHII & 0.5 & -1.4 & 2.4 & 0.7 & -1.2 & 2.6 & 1.1 & -0.9 & 3.1 \\
\hline \multicolumn{10}{|c|}{ DBP (mmHg) } \\
\hline Pooled & -0.3 & -1.3 & 0.6 & -0.3 & -1.2 & 0.7 & -0.2 & -1.2 & 0.9 \\
\hline SABRE & -0.2 & -1.8 & 1.3 & -0.3 & -1.9 & 1.3 & -0.2 & -2.0 & 1.6 \\
\hline WHII & -0.4 & -1.6 & 0.8 & -0.2 & -1.4 & 1.0 & 0.0 & -1.3 & 1.2 \\
\hline
\end{tabular}

a Model 1 adjusted for cohort (in pooled analyses), sex and age.

b Model 2 adjusted for cohort (in pooled analyses), sex, age, ethnicity, marital status and smoking.

c Model 3 adjusted for cohort (in pooled analyses), sex, age, ethnicity, marital status, smoking, area-level deprivation and NOx.

the models by sex as air pollution has been linked to greater progression of cIMT among females than males (Adar et al., 2013).

In addition to the pooled associations, fixed effects meta-analysis was performed based on Model 3 to investigate both cohort-specific and pooled estimates of the associations. Even though cIMT, SBP and DBP were approximately normally distributed, plots of residuals were examined to assess potential violations of linear regression assumptions. No violations were observed.

\section{Results}

Mean age of the total study population was 63 years $(S D=7)$, in the WHII cohort it was 61 years (SD =6), and in the SABRE cohort 69 years $(S D=6)$. Other descriptive statistics of the participants (pooled and by cohort) are presented in Table 1 . There were no differences in demographic variables and outcome variables between the cohort of all eligible participants and the cohort of participants without missing data. Distributions of night- and daytime road traffic noise, NOx and IMD scores, in the pooled sample and by study cohorts are presented in Table 2. In the pooled dataset, the correlation for night-time road noise and NOx was 0.33 . The correlation for daytime against nighttime road noise was 0.99 . Results using daytime noise were unsurprisingly comparable to results using night-time noise (Supplemental Tables 1-3) and are not discussed further.

In the pooled and cohort-specific linear regression analyses we observed non-significant positive associations between road noise exposure and cIMT. In the fully adjusted model (Model 3), cIMT increased by $9.1 \mu \mathrm{m}(95 \% \mathrm{CI}:-7.1,25.2)$ per $10 \mathrm{dBA}$ increase in night-time noise (Table 3). According to the fixed effects meta-analysis, a $10 \mathrm{dBA}$ increase in night-time noise was associated with an increased cIMT of $9.8 \mu \mathrm{m}(95 \% \mathrm{CI}:-6.0,25.6)$ (Fig. 1), which is comparable to the estimate from the pooled regression. Results for both cohorts were very similar. Sex-stratified results are presented in Supplemental Table 4. A slightly larger increase in cIMT per $10 \mathrm{dBA}$ increase in night-time noise were seen among females $(13.9 \mu \mathrm{m}, 95 \% \mathrm{CI}:-12.1,39.8)$ than among males $(8.2 \mu \mathrm{m}, 95 \% \mathrm{Cl}:-12.1,28.6)$.

Pooled association between night-time road noise and SBP was also positive although not statistically significant, the WHII cohort having a stronger indication of a positive association than SABRE (Table 3 and Fig. 2). Pooled and cohort-specific fully adjusted estimates for DBP were close to null (Table 3 and Fig. 2). The fixed effects meta-analysis resulted in similar effect estimates as the pooled analysis (Fig. 2). The sexstratified analyses for SBP and DBP did not provide evidence of a differential effect by gender (Supplemental Table 4). There were no pooled associations between $10 \mathrm{dBA}$ increase in night-time road noise and hypertension but with wide confidence intervals: odds ratio 0.98 (95\% CI: $0.78,1.23)$. The odds ratios from the fixed effects meta-analysis were similar (Supplemental Fig. 1). No significant associations were seen between NOx and any of cIMT, SBP or DBP when adjusted for the covariates and noise levels (Supplemental Table 5).

In analyses of noise by $5 \mathrm{dBA}$ categories, pooled mean differences in cIMT between participants exposed to night-time road noise levels 55$60 \mathrm{dBA}$ and $>60 \mathrm{dBA}$ vs. $<55 \mathrm{dBA}$ were $16.2 \mu \mathrm{m}(95 \% \mathrm{Cl}:-8.7,41.2)$ and $21.2 \mu \mathrm{m}(95 \% \mathrm{CI}:-2.5,44.9)$, respectively, after adjustment for the confounders (Table 4). Results were similar between cohorts (Table 4). A somewhat greater increase in cIMT was seen among females $(36.9 \mu \mathrm{m}$, $95 \% \mathrm{CI}: 0.1,73.7)$ than among males $(15.1 \mu \mathrm{m}, 95 \% \mathrm{CI}:-15.2,45.5)$ in the $>60 \mathrm{dBA}$ vs. $<55 \mathrm{dBA}$ night-noise category (Supplemental Table $6)$. Mean differences in SBP between participants exposed to nighttime road noise level 55-60 dBA and $>60 \mathrm{dBA}$ vs. $<55 \mathrm{dBA}$ were also positive $(1.1 \mathrm{mmHg}, 95 \% \mathrm{CI}-1.6,3.8$ and $0.1 \mathrm{mmHg}, 95 \% \mathrm{CI}:-2.5$, 2.6), but statistically non-significant (Table 4). Odds ratios for hypertension in the 55-60 dBA and $>60 \mathrm{dBA}$ vs. $<55 \mathrm{dBA}$ category were 0.86 (95\% CI: $0.60,1.21)$ and 0.94 (95\% CI: $0.68,1.32)$, respectively.

In the subset of participants not taking anti-hypertensive medications, the associations between night-time road noise levels and SBP

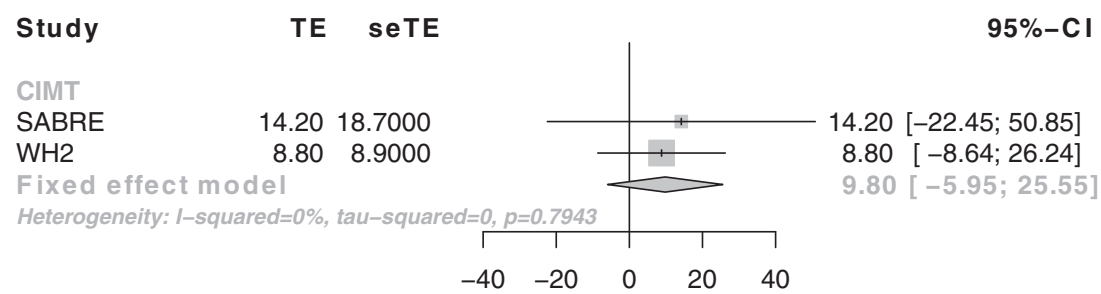

Fig. 1. Fixed effects meta-analysis for associations between $10 \mathrm{dBA}$ increase in night-time road traffic noise and carotid intima media thickness (CIMT). 


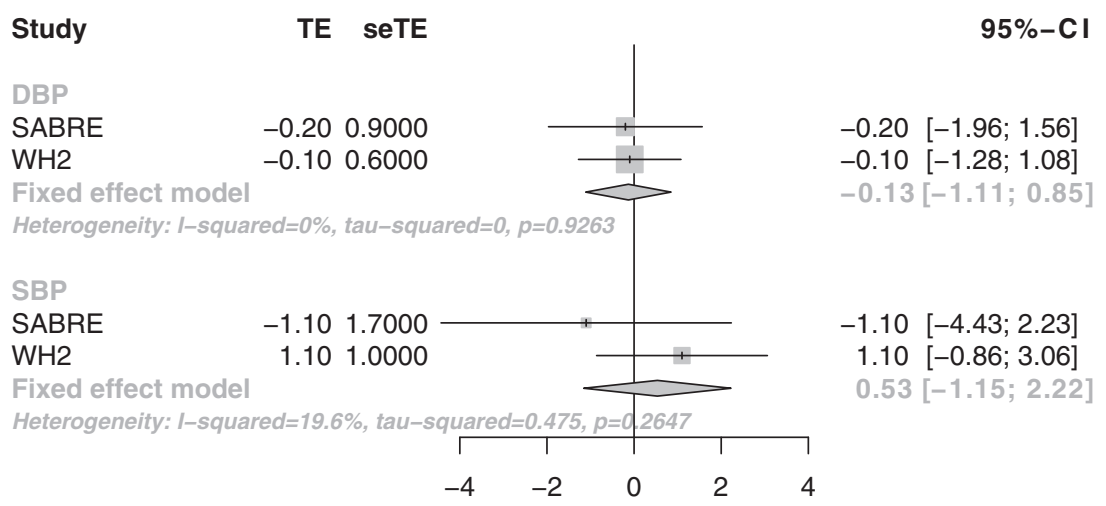

Fig. 2. Fixed effects meta-analysis for associations between $10 \mathrm{dBA}$ increase in night-time road traffic noise and diastolic (DBP) and systolic (SBP) blood pressure.

and DBP were mostly null. However, the association with cIMT was slightly more pronounced than in the overall cohort. In the fully adjusted model for the pooled sample (Model 3), cIMT increased by $14.1 \mu \mathrm{m}$ (95\% CI: - 3.1, 32.6) per $10 \mathrm{dBA}$ increase in night-time noise (Table 5 ). Mean differences in cIMT between these participants exposed to night-time noise levels 55-60 dBA and $>60 \mathrm{dBA}$ vs. $<55 \mathrm{dBA}$ were $16.9 \mu \mathrm{m}$ (95\% CI: $-10.7,44.5)$, and $32.6 \mu \mathrm{m}$ (95\% CI: 6.9, 59.0), respectively.

\section{Discussion}

This analysis using participants of two study cohorts from the Greater London, UK, found a positive association between long-term nighttime road traffic noise exposure and carotid intima-media thickness among a sub-set of non-medication users after adjustment for individual-level risk factors, air pollution and area-level deprivation. We observed no associations between traffic noise exposure and blood

Table 4

Pooled and cohort-specific mean differences in cIMT, SBP and DBP by night-time road traffic noise categories.

\begin{tabular}{|c|c|c|c|c|c|c|c|c|c|c|}
\hline \multirow[b]{2}{*}{ Night-time noise (dBA) } & \multirow[b]{2}{*}{$\mathrm{n}$} & \multicolumn{3}{|l|}{ Model $1^{\mathrm{a}}$} & \multicolumn{3}{|l|}{ Model $2^{\text {b }}$} & \multicolumn{3}{|l|}{ Model 3c } \\
\hline & & Estimate & $95 \% \mathrm{CI}$ & & Estimate & $95 \% \mathrm{CI}$ & & Estimate & $95 \% \mathrm{CI}$ & \\
\hline \multicolumn{11}{|l|}{ cIMT $(\mu \mathrm{m})$} \\
\hline \multicolumn{11}{|l|}{ Pooled } \\
\hline$<55 \mathrm{dBA}$ & 2187 & Ref & & & Ref & & & Ref & & \\
\hline $55-60 \mathrm{dBA}$ & 178 & 17.5 & -7.4 & 42.5 & 15.7 & -9.2 & 40.6 & 16.2 & -8.7 & 41.2 \\
\hline$>60 \mathrm{dBA}$ & 227 & 20.3 & -2.1 & 42.6 & 18.8 & -3.5 & 41.1 & 21.2 & -2.5 & 44.9 \\
\hline \multicolumn{11}{|l|}{ SABRE } \\
\hline$<55 \mathrm{dBA}$ & 491 & Ref & & & Ref & & & Ref & & \\
\hline $55-60 \mathrm{dBA}$ & 59 & 18.7 & -36.0 & 73.4 & 16.3 & -38.5 & 71.0 & 19.9 & -35.3 & 75.1 \\
\hline$>60 \mathrm{dBA}$ & 77 & 16.2 & -32.5 & 64.9 & 14.1 & -34.7 & 62.9 & 26.0 & -27.0 & 78.9 \\
\hline \multicolumn{11}{|l|}{ WHII } \\
\hline$<55 \mathrm{dBA}$ & 1696 & Ref & & & Ref & & & Ref & & \\
\hline $55-60 \mathrm{dBA}$ & 119 & 18.0 & -9.6 & 45.5 & 17.6 & -9.8 & 45.0 & 17.1 & -10.4 & 44.6 \\
\hline$>60 \mathrm{dBA}$ & 150 & 23.7 & -1.0 & 48.5 & 23.7 & -0.9 & 48.3 & 21.2 & -4.8 & 47.2 \\
\hline \multicolumn{11}{|l|}{ SBP (mmHg) } \\
\hline \multicolumn{11}{|l|}{ Pooled } \\
\hline$<55 \mathrm{dBA}$ & 2187 & Ref & & & Ref & & & Ref & & \\
\hline 55-60 dBA & 178 & 0.9 & -1.8 & 3.62 & 1.0 & -1.7 & 3.7 & 1.1 & -1.6 & 3.8 \\
\hline$>60 \mathrm{dBA}$ & 227 & -0.3 & -2.7 & 2.11 & -0.4 & -2.8 & 2.0 & 0.1 & -2.5 & 2.6 \\
\hline \multicolumn{11}{|l|}{ SABRE } \\
\hline$<55 \mathrm{dBA}$ & 491 & Ref & & & Ref & & & Ref & & \\
\hline 55-60 dBA & 59 & -1.1 & -6.2 & 4.0 & -1.7 & -6.8 & 3.3 & -1.7 & -6.7 & 3.4 \\
\hline$>60 \mathrm{dBA}$ & 77 & -2.1 & -6.7 & 2.5 & -2.8 & -7.3 & 1.7 & -2.7 & -7.6 & 2.2 \\
\hline \multicolumn{11}{|l|}{ WHII } \\
\hline$<55 \mathrm{dBA}$ & 1696 & Ref & & & Ref & & & Ref & & \\
\hline $55-60 \mathrm{dBA}$ & 119 & 2.0 & -1.2 & 5.2 & 2.3 & -0.9 & 5.5 & 2.4 & -0.8 & 5.6 \\
\hline$>60 \mathrm{dBA}$ & 150 & 0.6 & -2.3 & 3.5 & 0.8 & -2.1 & 3.6 & 1.4 & -1.6 & 4.4 \\
\hline \multicolumn{11}{|l|}{ DBP (mmHg) } \\
\hline \multicolumn{11}{|l|}{ Pooled } \\
\hline$<55 \mathrm{dBA}$ & 2187 & Ref & & & Ref & & & Ref & & \\
\hline $55-60 \mathrm{dBA}$ & 178 & -0.4 & -2.1 & 1.2 & -0.3 & -1.9 & 1.3 & -0.3 & -1.9 & 1.4 \\
\hline$>60 \mathrm{dBA}$ & 227 & -0.3 & -1.8 & 1.1 & -0.3 & -1.7 & 1.2 & -0.1 & -1.6 & 1.5 \\
\hline \multicolumn{11}{|l|}{ SABRE } \\
\hline$<55 \mathrm{dBA}$ & 491 & Ref & & & Ref & & & Ref & & \\
\hline $55-60 \mathrm{dBA}$ & 59 & -1.8 & -4.5 & 0.8 & -1.9 & -4.5 & 0.7 & -1.8 & -4.5 & 0.8 \\
\hline$>60 \mathrm{dBA}$ & 77 & -0.6 & -3.0 & 1.7 & -0.7 & -3.1 & 1.6 & -0.7 & -3.2 & 1.9 \\
\hline \multicolumn{11}{|l|}{ WHII } \\
\hline$<55 \mathrm{dBA}$ & 1696 & Ref & & & Ref & & & Ref & & \\
\hline $55-60 \mathrm{dBA}$ & 119 & 0.4 & -1.6 & 2.4 & 0.6 & -1.4 & 2.6 & 0.6 & -1.4 & 2.6 \\
\hline$>60 \mathrm{dBA}$ & 150 & -0.2 & -2.0 & 1.6 & -0.1 & -1.8 & 1.7 & 0.3 & -1.6 & 2.2 \\
\hline
\end{tabular}

a Model 1 adjusted for cohort (in pooled analyses), sex and age.

b Model 2 adjusted for cohort (in pooled analyses), sex, age, ethnicity, marital status and smoking.

c Model 3 adjusted for cohort (in pooled analyses), sex, age, ethnicity, marital status, smoking, area-level deprivation and NOx. 
pressure or hypertension. Differences between the pooled and metaanalysis estimates were small, supporting the generalizability of our findings.

To our knowledge, this is the first study to examine associations between road traffic noise exposure and cIMT. Some previous studies have examined road proximity in relation to intermediate cardiovascular markers, which may reflect both noise and air pollution exposures. One earlier cross-sectional study examined coronary artery calcification (CAC) and found 1.63 times higher odds of having a high amount of CAC among individuals living $\leq 50 \mathrm{~m}$ compared to those living $>200 \mathrm{~m}$ from a major road (Hoffmann et al., 2009). Studies with longitudinal data have suggested that living within $100 \mathrm{~m}$ from a highway is associated with progression of atherosclerosis, indicated as an increase in cIMT (Kunzli et al., 2010), and long-term exposure to black carbon, another proxy for exposure to traffic, has been linked to an increase in cIMT in elderly men (Wilker et al., 2013). In the WHII cohort, increase in particulate matter has also been associated with extent of subclinical atherosclerosis measured by cIMT (Tonne et al., 2012). However, in these studies traffic noise was not measured or included as a separate exposure variable. Although positive associations between particulate air pollution and cIMT have been reported (Tonne et al., 2012; Wilker et al., 2013), inverse non-significant associations were observed with $\mathrm{NO}_{2}$ or NOx in a study with four European cohorts (Perez et al., 2015), which agrees with our findings.

We did not observe robust associations between long-term exposure to roadtraffic noise and systolic or diastolic blood pressure, or hypertension. Previous studies, including a meta-analysis of 24 studies, have shown associations between traffic noise and hypertension (Babisch et al., 2014; Barcelo et al., 2016; van Kempen et al., 2012), but other studies have not (Sorensen et al., 2011) or found that associations lost statistical significance after adjustment for air pollution (Babisch et al., 2014). Results for blood pressure have been less frequently reported. Similarly to our findings, studies on the long-term effects of traffic noise exposure on SBP or DBP have reported no (Dratva et al., 2011; Fuks et al., 2011) or weak associations (Sorensen et al., 2011). Two possible reasons for this are exposure misclassification and modifying effect of medication, discussed below. One study that used nighttime traffic noise exposure estimated at the façade of the participants' residences suggested a positive association with SBP and DBP. They also reported that night-time traffic noise exposure estimated for indoors was associated with increased SBP (Foraster et al., 2014). This suggests that exposure misclassification may exist when using only outdoor traffic noise estimates, which attenuates the possible associations. In this study, we had no information about the direction of the bedroom window in relation to the streets. It has also been suggested that high correlation ( 0.7 or higher) between noise and an air pollutant used as a covariate would have prevented researchers from seeing associations between noise and blood pressure in previous studies (Foraster et al., 2014). In our data correlation between road traffic noise and NOx was around 0.30 , indicating that this cannot explain the weak findings. Also, other studies have suggested noise is an independent contributor to health risks (Stansfeld, 2015). Overall, it is more difficult to assess a relationship between traffic noise and blood pressure, than hypertension, given the modifying effect of medication. Although we, and others (Dratva et al., 2011; Foraster et al., 2014; Fuks et al., 2011), attempted to adjust for the medication use, this may be another possible reason resulting in the lack of a clear association for blood pressure.

We observed a positive non-significant association between nighttime road traffic noise and cIMT that was slightly larger in the subset of participants not taking anti-hypertensive medications, and slightly larger among females than males, which agrees with findings for particulate air pollutants and cIMT (Adar et al., 2013). The small numbers of participants exposed to noise levels above $60 \mathrm{dBA}$ may, however, have impaired our ability to detect significant associations. This relates to the use of postcode- and not address-level noise estimates that might have resulted in reduced exposure contrasts limiting statistical power. Nevertheless, we were able to conduct a small study using the TRAFFIC study (King's College London, 2014) estimates of day-time road traffic noise levels within the CHASE cohort (Whincup et al., 2010), covering most of the geographical area related to cohorts in this study, to assess the difference between postcode and address level noise estimates ( $n=4692 ; ~ 2.5 \%$ of all postcodes). For $85 \%$ of the addresses, the differences between postcode and address level noise estimates were smaller or equal to $1 \mathrm{dBA}$. The differences were bigger than $3 \mathrm{dBA}$ for only $3 \%$ of the addresses. Although pooling data increased the sample size and the heterogeneity in the noise range covered, which had a favourable impact on power, statistical power remained as an important limitation. In addition, the exposure model used is likely to have resulted in some exposure misclassification, with exposure at low noise levels being over-estimated and exposure in areas with heavily trafficked minor roads underestimated. Therefore, we used not only linear but also categorical exposure metrics which may have reduced the misclassification. Our study did not include noise from rail and aircraft traffic as in London these have different distributions from road traffic noise (Carey et al., 2016; Halonen et al., 2015a; Hansell et al., 2013). Rail and aircraft noise also have different acoustic characteristics and thus may not be simply additive with road noise (Basner et al., 2014). Another limitation is that we did not have information on residential history for all

Table 5

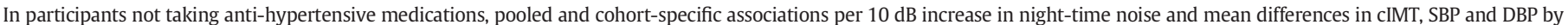
night-time road traffic noise categories in model $3^{\mathrm{a}}$.

\begin{tabular}{|c|c|c|c|c|c|c|c|c|c|c|}
\hline & \multirow[b]{2}{*}{$\mathrm{n}$} & \multicolumn{3}{|c|}{$\operatorname{cIMT}(\mu \mathrm{m})$} & \multicolumn{3}{|c|}{$\mathrm{SBP}(\mathrm{mmHg})$} & \multicolumn{3}{|c|}{ DBP (mmHg) } \\
\hline & & Estimate & $95 \% \mathrm{CI}$ & & Estimate & $95 \% \mathrm{CI}$ & & Estimate & $95 \% \mathrm{CI}$ & \\
\hline \multicolumn{11}{|l|}{ Continuous } \\
\hline Pooled & 1702 & 14.7 & -3.1 & 32.6 & 0.4 & -1.6 & 2.3 & -0.2 & -1.5 & 1.0 \\
\hline SABRE & 197 & 25.1 & -29.9 & 80.2 & 0.0 & -5.1 & 5.1 & -0.6 & -3.2 & 2.1 \\
\hline WHII & 1505 & 12.4 & -6.6 & 31.4 & 0.7 & -1.4 & 2.8 & 0.0 & -1.4 & 1.4 \\
\hline \multicolumn{11}{|l|}{ Categorical } \\
\hline$<55 \mathrm{dBA}$ & 1444 & Ref & & & Ref & & & Ref & & \\
\hline $55-60 \mathrm{dBA}$ & 114 & 16.9 & -10.7 & 44.5 & 2.0 & -1.0 & 5.0 & 0.4 & -1.6 & 2.3 \\
\hline$>60 \mathrm{dBA}$ & 144 & 32.6 & 6.2 & 59.0 & -0.4 & -3.2 & 2.5 & -0.4 & -2.2 & 1.5 \\
\hline \multicolumn{11}{|l|}{ SABRE } \\
\hline$<55 \mathrm{dBA}$ & 148 & Ref & & & Ref & & & Ref & & \\
\hline $55-60 \mathrm{dBA}$ & 20 & 45.0 & -36.3 & 126.3 & -1.4 & -9.0 & 6.1 & -3.9 & -7.8 & -0.1 \\
\hline$>60 \mathrm{dBA}$ & 29 & 36.8 & -39.5 & 113.1 & -0.7 & -7.7 & 6.4 & -0.2 & -3.8 & 3.4 \\
\hline \multicolumn{11}{|l|}{ WHII } \\
\hline$<55 \mathrm{dBA}$ & 1296 & Ref & & & Ref & & & Ref & & \\
\hline $55-60 \mathrm{dBA}$ & 94 & 12.2 & -17.3 & 41.8 & 3.0 & -0.3 & 6.3 & 1.3 & -0.9 & 3.5 \\
\hline$>60 \mathrm{dBA}$ & 115 & 32.6 & 4.3 & 60.9 & -0.1 & -3.2 & 3.0 & -0.3 & -2.4 & 1.8 \\
\hline
\end{tabular}

a Model 3 adjusted for cohort (in pooled analyses), sex, age, ethnicity, marital status, smoking, area-level deprivation and NOx. 
participants, therefore exposure of those participants who had lived in the residential postcode area used for the exposure linkage for a short time period may have been misclassified. As in all epidemiological noise studies to date, information on daily activities of the participants was not available and was not accounted for in the exposure modelling, which may also have caused some exposure misclassification. However, in this study, we used the same road traffic noise model as in our previous ecological study on cardiovascular morbidity and mortality in London (Halonen et al., 2015b). The strengths of this study also include use of individual-level information on cIMT, blood pressure and hypertension from two London cohorts, as well as control for possible individual- and area-level confounders such as smoking, area-level deprivation and air pollution.

\section{Conclusions}

Our findings from Greater London suggest that after taking into account potential confounders, such as air pollution, the association between night-time road traffic noise and cIMT is only observed among persons who do not use antihypertensive medication, and the associations with blood pressure and hypertension are largely null. Thus, we found only weak evidence to support the hypothesis that associations between road traffic noise and stroke are via the cIMT or hypertension pathway. To confirm these findings, the associations between road traffic noise exposure and cIMT should be further examined in longitudinal settings with large study samples and in locations with more variation in the noise exposure levels.

\section{Funding}

This work was supported by the UK Natural Environment Research Council, Medical Research Council, Economic and Social Research Council, Department of Environment, Food and Rural Affairs, and Department of Health (NE/I007806/1, NE/I00789X/1, NE/I008039/1) through the cross-research council Environmental Exposures \& Health Initiative. The work of the UK Small Area Health Statistics Unit (SAHSU) is funded by Public Health England as part of the MRC-PHE Centre for Environment and Health, and by the UK Medical Research Council (K013351). The UK Medical Research Council, British Heart Foundation, and the US National Institutes of Health (R01HL36310, R01AG013196) have supported collection of data in the Whitehall II Study and the work by MK. The SABRE study was supported by a joint programme grant from the Welcome Trust and British Heart Foundation.

\section{Conflict of interest}

None declared.

\section{Acknowledgement}

We thank all participating women and men in the Whitehall II Study and the SABRE study, as well as all Whitehall II research scientists, study and data managers and clinical and administrative staff and the SABRE study team who made the study possible.

\section{Appendix A. Supplementary data}

Supplementary data to this article can be found online at http://dx. doi.org/10.1016/j.envint.2016.09.023.

\section{References}

Adar, S.D., et al., 2013. Fine particulate air pollution and the progression of carotid intimamedial thickness: a prospective cohort study from the multi-ethnic study of atherosclerosis and air pollution. PLoS Med. 10, e1001430.
Babisch, W., 2014. Updated exposure-response relationship between road traffic noise and coronary heart diseases: a meta-analysis. Noise Health 16, 1-9.

Babisch, W., et al., 2014. Associations between traffic noise, particulate air pollution, hypertension, and isolated systolic hypertension in adults: the KORA study. Environ. Health Perspect. 122, 492-498.

Barcelo, M.A., et al., 2016. Long term effects of traffic noise on mortality in the city of Barcelona, 2004-2007. Environ. Res. 147, 193-206.

Basner, M., et al., 2014. Auditory and non-auditory effects of noise on health. Lancet 383 1325-1332.

Beelen, R., et al., 2013. Development of NO2 and NOx land use regression models for estimating air pollution exposure in 36 study areas in Europe - the ESCAPE project. Atmos. Environ. 72, 10-23.

Beevers, S.D., et al., 2013. Air pollution dispersion models for human exposure predictions in London. J. Expo. Sci. Environ. Epidemiol. 23, 647-653.

Bodin, T., et al., 2016. Road traffic noise, air pollution and myocardial infarction: a prospective cohort study. Int. Arch. Occup. Environ. Health 89, 793-802.

Carey, I.M., et al., 2016. Traffic pollution and the incidence of cardiorespiratory outcomes in an adult cohort in London. Occup. Environ. Med.

Cui, J.S., et al., 2003. Antihypertensive treatments obscure familial contributions to blood pressure variation. Hypertension 41, 207-210.

Dratva, J., et al., 2011. Transportation noise and blood pressure in a population-based sample of adults. Environ. Health Perspect. 120, 50-55.

Eikendal, A.L., et al., 2015. Common carotid intima-media thickness relates to cardiovascular events in adults aged $<45$ years. Hypertension $65,707-713$.

Elliott, P., et al., 2005. Primary prevention of high blood pressure. In: Marmot, M., Elliott, P. (Eds.), Coronary Heart Disease Epidemiology. From Aetiology to Public Health. Oxford University Press.

Foraster, M., et al., 2014. High blood pressure and long-term exposure to indoor noise and air pollution from road traffic. Environ. Health Perspect. 122, 1193-1200.

Fuks, K., et al., 2011. Long-term urban particulate air pollution, traffic noise, and arterial blood pressure. Environ. Health Perspect. 119, 1706-1711.

Gulliver, J., et al., 2015. Development of an open-source road traffic noise model for exposure assessment. Environ. Model. Softw. 74, 183-193.

Halonen, J.I., et al., 2015a. Is long-term exposure to traffic pollution associated with mortality? A Small-area Study in London. Environmental Pollution (Barking, Essex : 1987) Epub ahead of print

Halonen, J.I., et al., 2015b. Road traffic noise is associated with increased cardiovascular morbidity and mortality and all-cause mortality in London. Eur. Heart J. 36, 2653-2661.

Hansell, A.L., et al., 2013. Aircraft noise and cardiovascular disease near Heathrow airport in London: small area study. BMJ 347, f5432.

Hoffmann, B., et al., 2009. Residential traffic exposure and coronary heart disease: results from the Heinz Nixdorf Recall Study. Biomarkers 14, 74-78.

Jennings, J.R., et al., 2004. Exaggerated blood pressure responses during mental stress are prospectively related to enhanced carotid atherosclerosis in middle-aged Finnish men. Circulation 110, 2198-2203.

King's College London, 2014. Traffic project. http://www.kcl.ac.uk/biohealth/research/ divisions/aes/research/ERG/research-projects/traffic/index.aspx.

Kivimaki, M., et al., 2009. Validating the Framingham Hypertension Risk Score: results from the Whitehall II study. Hypertension 54, 496-501.

Kunzli, N., et al., 2010. Ambient air pollution and the progression of atherosclerosis in adults. PLoS One 5, e9096.

Marmot, M., et al., 2005. Cohort profile: the Whitehall II study. Int. J. Epidemiol. 34 251-256.

Martin, U., et al., 2015. Ethnicity and differences between clinic and ambulatory blood pressure measurements. Am. J. Hypertens. 28, 729-738.

McLennan, D., et al., 2011. The English Indices of Deprivation 2010. Department of Communities and Local Government, London.

Messner, B., et al., 2014. Smoking and cardiovascular disease: mechanisms of endothelia dysfunction and early atherogenesis. Arterioscler. Thromb. Vasc. Biol. 34, 509-515.

Monrad, M., et al., 2016. Residential exposure to traffic noise and risk of incident atrial fibrillation: a cohort study. Environ. Int. 92-93, 457-463.

Munzel, T., et al., 2014. Cardiovascular effects of environmental noise exposure. Eur. Heart J. 35, 829-836.

Munzel, T., et al., 2016. Environmental stressors and cardio-metabolic disease: part I-epidemiologic evidence supporting a role for noise and air pollution and effects of mitigation strategies. Eur. Heart J.

Noble, M., et al., 2004. The English Indices of Deprivation 2004 (Revised). Office of the Deputy Prime Minister, London.

Office for National Statistics, 2014. Super output areas (SOAs). http://www.ons.gov.uk/ ons/guide-method/geography/beginner-s-guide/census/super-output-areas-soas-/ index.html.

O'Leary, D.H., et al., 1999. Carotid-artery intima and media thickness as a risk factor for myocardial infarction and stroke in older adults. Cardiovascular Health Study Collaborative Research Group. N. Engl. J. Med. 340, 14-22.

Perez, L., et al., 2015. Air pollution and atherosclerosis: a cross-sectional analysis of four European cohort studies in the ESCAPE study. Environ. Health Perspect. 123 597-605.

Sorensen, M., et al., 2011. Exposure to road traffic and railway noise and associations with blood pressure and self-reported hypertension: a cohort study. Environ. Health 10, 92.

Stansfeld, S.A., 2015. Noise effects on health in the context of air pollution exposure. Int J. Environ. Res. Public Health 12, 12735-12760.

Tillin, T., et al., 2012. Southall and Brent REvisited: cohort profile of SABRE, a UK population-based comparison of cardiovascular disease and diabetes in people of European, Indian Asian and African Caribbean origins. Int. J. Epidemiol. 41, 33-42. 
Tonne, C., et al., 2012. PM mass concentration and PM oxidative potential in relation to carotid intima-media thickness. Epidemiology 23, 486-494.

van Kempen, E., et al., 2012. The quantitative relationship between road traffic noise and hypertension: a meta-analysis. J. Hypertens. 30, 1075-1086.

Whincup, P.H., et al., 2010. Early emergence of ethnic differences in type 2 diabetes precursors in the UK: the Child Heart and Health Study in England (CHASE Study). PLoS Med. 7, e1000263.
WHO, 2011. Burden of disease from environmental noise. Quantification of Healthy Life Years Lost in Europe. Germany WHO Regional Office for Europe, Bonn.

Wilker, E.H., et al., 2013. Long-term exposure to black carbon and carotid intima-media thickness: the normative aging study. Environ. Health Perspect. 121, 1061-1067. 